\title{
Synthesis of (3-Cyano-4, 5, 6, 7-Tetrahydrobenzo [B] Thiophen-2- Yl) Formamidine Derivatives as Effective Antimicrobial Agents
}

\author{
Poonam S. Patil ${ }^{1}$, Satish M. Chavan ${ }^{1,2}$, Raghunath B. Toche ${ }^{1 *}$, Avinash D. \\ Bhole $^{3}$, Vasant M.Patil ${ }^{1}$, Pankaj B.Aware ${ }^{1}$ \\ ${ }^{1}$ Organic Chemistry Research Center, Department of Chemistry ${ }^{1}$ and Department of Microbiology ${ }^{3}$ K.R.T. Arts, \\ B.H. Commerce and A. M. Science College, Shivajinagar, Gangapur Road, Nashik- 422 002, (MS), India. \\ ${ }^{2}$ Department of Chemistry, R.N.C. Arts, J.D.B. Commerce and N.S.C. Science College, Nashik-Road, Nashik- \\ 422 101, (MS), India
}

\begin{abstract}
Series of tetrahydrobenzo[b]thiophen-2-yl)-N, N-dimethylformamidine derivatives were synthesized from 2-amino-4, 5, 6,7-tetrahydrobenzo[b]thiophene-3-carbonitrile. The new derivatives were checked good antimicrobial activities against $S$. aureus, E. coli and C. albicans, and A. Niger microbes.
\end{abstract}

Keywords: Dimethylformamidine, 2-amino-4, 5, 6, 7-tetrahydrobenzo[b]thiophene-3-carbonitrile Antimicrobial activity

\section{Introduction}

Substituted benzothiophene derivatives are important heterocyclic compounds found in many natural compounds. These derivatives have showed number of biological activities hence used in pharmaceuticals, agrochemicals and for diagnostics, optoelectronics, dyes etc. [1]. They had been reported as anti-tubercular [2], anti-inflammatory [3], anti-microbial [4,12], antianxiety [5] agents. Literature survey revealed that substituted benzothiophene derivatives are potent and selective inhibitor of human leukocyte elastase [6], kinesin spindle protein (KSP) [7], tubulin [8] and tyrosine kinase of the fibroblast growth factor receptors (FGRF) [9] as well as adenosine A1 receptor allosteric enhancers [10]. K. Madhavi prepared 4, 5, 6, 7-tetrahydrobenzo[b] thiophene-3-carboxylate derivatives and evaluated their antioxidant, antibacterial activities [11, 13]. 2-Amino-3$N$-(propylcarboxamido)- 4, 5, 6,7-tetrahydrobenzo[b]thiophene-3-carboxylate showed mycolytic activities [12] which resulted in marketing as antifungal agents sertaconazol. $\mathrm{N}$-ethoxymethino derivatives, $\mathrm{N}$ phenylaminomethino derivatives, hydrazine derivatives, pyrazole derivatives, and $N$-methinonitrilo derivatives of 4,5,6,7-tetrahydrobenzo[b]thiophene were synthesized and cytotoxic and anti-leshmanial activity [13] was evaluated. Spiro(benzothieno[2,3- $d$ ] pyrimidine-4-ones, benzothinenopyrimidine derivatives, triazolo and imidazolo pyrimidine derivatives and 3-amino-(2-alkylamino)benzothie- nopyrimidine-4,5-dione derivatives of 3 -aminobenzothieno[2,3- $d$ ] pyrimidine showed antiinflammetry, analgesic and ulcerogenic activities. Mohamed Said and co-workers synthesized thieno (3,2- $d$ )- (1,2,3)-triazines and $N$-(3-cyano-5,6-dihydro- $4 H$-cyclopenta (b) thiophenes and evaluated their anticancer activity [15]. The reaction involving bis-nucleophilic and biselectrophilic attack, dipolar cyclization and condensation furnished targeted products. The wide application and diverse synthetic procedures promoted many chemists to design and synthesize these classes of bioactive heterocycles [16-22].

In this paper we have concentrated our efforts towards the synthesis of 3/4-substituted phenyl- $N^{\prime}-(3-$ cyano-4,5,6,7-tetrahydrobenzo[b]thiophen-2-yl)formamidines 7. 3/4-substituted phenyl)- $N^{\prime}$-(3-cyano-4,5,6,7tetrahydrobenzo[b]thiophen-2-yl)formamidines $\mathbf{7 a - h}$ showed good antimicrobial activities as compared with Gentamicin and Fluconazole.

\section{Materials and methods}

The physical constants (melting point) of all new compounds were recorded on Gallencamp melting point equipment (Model no MFB-595) using open capillary tubes and uncorrected. IR spectra of the compounds were recorded on Bruker FTIR-TENSOR-II. ${ }^{1} \mathrm{HNMR}$ spectra of the compounds were recorded on Bruker Avance II $(500 \mathrm{MHz}) \mathrm{NMR}$ instrument. The $\mathrm{CDCl}_{3}$ or DMSO was used to record the NMR using TMS (Tetramethylsilane) as internal standard. Chemical shifts are given in $\delta$ ppm and splitting of NMR samples are given as singlet (s), broad singlet (bs), doublet (d), triplet (t), multiplets (m).The reactions were monitored on thin layer chromatography (TLC $0.2 \mathrm{~mm}$ silica gel $60 \mathrm{~F}_{254}$ Merck plates) plates using UV light 254 and $366 \mathrm{~nm}$. The chemicals were purchased from Sd Fine chemicals, Sigma Aldrich, Merck, Loba chemie and used without purification while solvents were purified by standard literature protocols. 


\section{Result And Discussion}

Compound 3 was synthesized by Gewald thiophene synthesis [23-25] using cyclohexanone, sulfur and malononitrile in presence of catalytic amount of morpholine in ethanol at reflux temperature. The structure was established on the basis of IR, ${ }^{1} H$ NMR data and compared with the literature M.P.

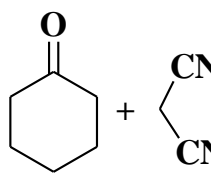

1

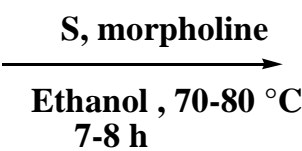<smiles>N#Cc1c(N)sc2c1CCCC2</smiles>

$380 \%$

Scheme 1 2-Amino-4,5,6,7-tetrahydrobenzo[b]thiophene-3-carbonitrile (3)

The key intermediate 4,5,6,7-tetrahydrobenzo [b]thiophen-2-yl)- $N, N$-dimethylformamidine 5 was obtained by reacting 2-amino-4,5,6,7-tetrahydrobenzo [b]thiophene-3-carbonitrile 3 with DMF/DMA (Scheme 2). This compound was characterized by IR, ${ }^{1} \mathrm{H}$ NMR data. For e.g. IR spectrum showed stretching frequencies at $1613,2288 \mathrm{~cm}^{-1}$ was assigned to $\mathrm{C}=\mathrm{N}$ and $\mathrm{CN}$. The ${ }^{1} \mathrm{H}$ NMR spectrum of 5 showed triplets at $\delta 1.82$ and 2.52 for $2 \times \mathrm{CH}_{2}-\mathrm{CH}_{2}$, singlet at 7.66, for $=\mathrm{CH}$ singlet at $\delta 3.07$ assigned $\mathrm{CH}_{3}$ group. The formidine 5 on reacting with glycine using catalytic amount of glacial acetic acid in ethanol afforded $N^{\prime}$-(3-cyano-4,5,6,7-tetrahydrobenzo[b] thiophen-2-yl)-2-((E) formamido) acetic acid 7a. Similar reaction of compound $\mathbf{5}$ when reacted with aromatic amines yielded (3/4-substituted phenyl)- $N$ '-(3-cyano-4,5,6,7-tetrahydrobenzo[b]thiophen-2-yl)formamidines 7bh with $60-80 \%$ yields. (Scheme 3 )<smiles>N#Cc1c(N)sc2c1CCCC2</smiles>

3<smiles>COC(OC)N(C)C</smiles>

4

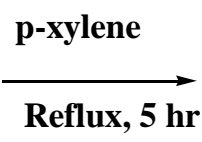

$5 \quad 78 \%$

Scheme 2 (E)-N'-(3-cyano-4,5,6,7-tetrahydrobenzo [b]thiophen-2-yl)-N,N-dimethyl-formamidine (5)

The compound 7a was characterized as the IR spectrum of $7 \mathbf{a}$ showed stretching frequencies at 2198, $2935,1766,1620 \mathrm{~cm}^{-1}$ for $\mathrm{CN}, \mathrm{CH}, \mathrm{C}=\mathrm{N}, \mathrm{C}=\mathrm{C} \mathrm{cm}^{-1}$ respectively. The PMR of this compound showed singlet at $\delta 10.9$, triplet at $\delta 1.85-2.59$, quartet at $\delta 2.50$, doublet at $\delta 7.67$ and doublet at $\delta 3.10$ were assigned to $\mathrm{OH}_{1} \mathrm{CH}_{2}-$ $\mathrm{CH}_{2}, \mathrm{NH}, \mathrm{CH}, \mathrm{CH}_{2}$ groups respectively.

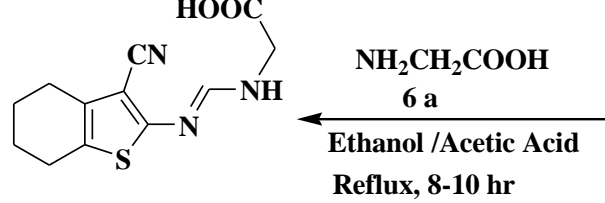

$7 a$

$80 \%$<smiles>CN=Cc1sc2c(c1C#N)CCCC2</smiles><smiles>CN(C)C=Nc1sc2c(c1C#N)CCCC2</smiles>

Scheme 3 Synthesis of $N^{\prime}$-(3-cyano-4,5,6,7-tetrahydrobenzo[b]thiophen-2-yl)-2-((E)formamido)acetic acid, (7a) and (E)- $N$-(aryl) (3-cyano-4,5,6,7-tetrahydrobenzo[b]thiophen-2-yl)-formamidine derivatives (7b-h)

\begin{tabular}{|c|l|c|}
\hline Compound & Amine & \% Yield \\
\hline $7 \mathbf{a}$ & Glycine & $\mathbf{8 0}$ \\
\hline $7 \mathbf{b}$ & Aniline & 68 \\
\hline $7 \mathbf{c}$ & 4-Chloroaniline & 62 \\
\hline $7 \mathbf{d}$ & 4-Bromoaniline & $\mathbf{6 1}$ \\
\hline $7 \mathbf{e}$ & 4- Nitroaniline & $\mathbf{7 0}$ \\
\hline $\mathbf{7 f}$ & 3- Chloroaniline & $\mathbf{6 0}$ \\
\hline $7 \mathbf{g}$ & 3- Nitroaniline & $\mathbf{7 5}$ \\
\hline $7 \mathbf{h}$ & 2-Aminopyridine & $\mathbf{6 8}$ \\
\hline
\end{tabular}

Antimicrobial activity: 
The antimicrobial activity of newly synthesized compounds (7a-h) was determined against E.coli (Gram+ve), S.aureus (Gram-ve) bacteria and fungi such as A. niger, C. albicans. The antimicrobial activity evaluation was performed using fungi reseeded in Crazek Dox agar for $48 \mathrm{hr}$ at $25{ }^{\circ} \mathrm{C}$ and bacteria reseeded in Muller-Hinton broth for $24 \mathrm{hr}$ at $37{ }^{\circ} \mathrm{C}$. The standard strains required for antimicrobial assay was obtained from Microbial Culture Collection Centre, Pune, Maharashtra, India. The antimicrobial activities of tested samples were carried out in triplicate against E.coli (Gram+ve), S.aureus (Gram-ve) bacteria and fungi such as A.niger, C. albicans.

Table 1: Antimicrobial screening of compounds (7a-h): Inhibition Zone Diameter ( $\mathrm{mm}$ )

\begin{tabular}{|c|l|c|c|c|c|}
\hline Compound & Amine & $\begin{array}{c}\text { E. coli } \\
\text { ATCC25922 }\end{array}$ & $\begin{array}{c}\text { S. aureus } \\
\text { ATCC29737 }\end{array}$ & $\begin{array}{c}\text { A.niger } \\
\text { MCIM 545 }\end{array}$ & $\begin{array}{c}\text { Calbicans } \\
\text { MTCC 277 }\end{array}$ \\
\hline 7a & Glycine & $13 \pm 0.8$ & $14 \pm 1.2$ & $13 \pm 0.6$ & $14 \pm 0.8$ \\
\hline 7b & Aniline & $15 \pm 1.1$ & $16 \pm 0.7$ & $17 \pm 1.1$ & $16 \pm 0.8$ \\
\hline 7c & Glycine & $16 \pm 0.8$ & $16 \pm 0.8$ & $18 \pm 0.5$ & $18 \pm 0.9$ \\
\hline 7d & Aniline & $17 \pm 0.8$ & $18 \pm 0.3$ & $17 \pm 0.7$ & $18 \pm 0.4$ \\
\hline 7e & 4-Chloroaniline & $15 \pm 1.1$ & $16 \pm 0.6$ & $17 \pm 0.3$ & $16 \pm 0.7$ \\
\hline 7f & 4-Bromoaniline & $14 \pm 0.9$ & $15 \pm 1.1$ & $16 \pm 1.2$ & $15 \pm 0.8$ \\
\hline 7g & 4- Nitroaniline & $18 \pm 0.8$ & $17 \pm 0.4$ & $14 \pm 1.1$ & $15 \pm 1.3$ \\
\hline 7h & 3- Chloroaniline & $12 \pm 0.6$ & $13 \pm 0.5$ & $12 \pm 0.6$ & - \\
\hline \multicolumn{7}{|l|}{ DMSO } & $11 \pm 0.7$ & $12 \pm 0.9$ & $23 \pm 0.3$ \\
\hline
\end{tabular}

DMSO was loaded negative control. The solutions of the test samples were prepared in DMSO in desired concentrations of 40, 20,10 $\mu \mathrm{g} / \mathrm{mL}$. The Gentamicin $(10 \mu \mathrm{g} / \mathrm{mL})$ and Fluconazole $(20 \mu \mathrm{g} / \mathrm{mL}) \mathrm{were}$ used as standards for antimicrobial activity evaluation. The zone of inhibition ( $\mathrm{mm})$ was measured as per National Committee for Chemical Laboratory Standards (NCCLS, M7-A5, January 2000). The antimicrobial activity of 3- nitroaniline was more than 4-chloroaniline and 4- bromoaniline.

The compound $\mathbf{7 g}$ exhibited excellent antibacterial activities against Gram+ve and Gram-ve bacteria viz. Staphylococcus aureus, Escherichia coli with MIC $10 \mu \mathrm{g} / \mathrm{mL}$ due to presence of p-nitro group. Also compound $7 \mathrm{~g}$ showed excellent antifungal activities against Aspergillus niger and Candida albicans with MIC $10 \mu \mathrm{g} / \mathrm{mL}$ may be due to presence of nitro group. The compound $\mathbf{7 c}$ and $\mathbf{7 d}$ showed moderate antibacterial activity against Escherichia coli with MIC $20 \mu \mathrm{g} / \mathrm{mL}$ when compared with Gentamicin. The compounds 7c, 7d, 7e and 7f showed excellent antifungal activities against Aspergillus niger (MCIM 545). Similarly, compounds 7b, 7c, 7d showed equivalent antifungal activities against Candida albicans with MIC $20 \mu \mathrm{g} / \mathrm{mL}$ as compared with Fluconazole.

Table 2: Antimicrobial screening of compounds (7a-h): MIC in $\mu \mathrm{g} / \mathrm{mL}$ values

\begin{tabular}{|c|c|c|c|c|c|}
\hline Compd & Amine & $\begin{array}{c}\text { E. coli } \\
\text { ATCC25922 } \\
\end{array}$ & $\begin{array}{c}\text { S. aureus } \\
\text { ATCC29737 }\end{array}$ & $\begin{array}{c}\text { A.niger } \\
\text { MCIM 545 }\end{array}$ & $\begin{array}{l}\text { C. albicans } \\
\text { MTCC } 277\end{array}$ \\
\hline $7 \mathbf{a}$ & Glycine & 80 & 40 & 80 & 40 \\
\hline $7 \mathbf{b}$ & Aniline & 40 & 40 & 20 & 20 \\
\hline 7c & Glycine & 20 & 20 & 20 & 20 \\
\hline $7 d$ & Aniline & 20 & 20 & 20 & 20 \\
\hline $7 e$ & 4-Chloroaniline & 40 & 20 & 20 & 40 \\
\hline $7 f$ & 4-Bromoaniline & 40 & 40 & 20 & 40 \\
\hline $7 g$ & 4- Nitroaniline & 10 & 10 & 10 & 10 \\
\hline \multirow[t]{3}{*}{$7 \mathrm{~h}$} & 3- Chloroaniline & 80 & 80 & 40 & 40 \\
\hline & Gentamicin & 10 & 10 & - & - \\
\hline & Fluconazole & - & - & 20 & 20 \\
\hline
\end{tabular}

\section{Experimental}

2-Amino-4,5,6,7-tetrahydrobenzo[b]thiophene-3-carbonitrile (3)

The mixture of cyclohexanone $(0.01 \mathrm{~mol})$ and malononitrile $(0.01 \mathrm{~mol}, 0.66 \mathrm{~g})$ was stirred in ethanol at $50{ }^{\circ} \mathrm{C}$ for $30 \mathrm{~min}$, after $30 \mathrm{~min}$ elemental sulfur $(0.02 \mathrm{~mol})$ was added portion wise. Then morpholine $(0.01 \mathrm{~mL})$ was added to reaction mixture drop wise. The resulting reaction mass was stirred further $7-8 \mathrm{~h}$ in an oil bath at $70-80{ }^{\circ} \mathrm{C}$ (TLC check, toluene: acetone, 9:1, v/v). After completion of reaction, reaction mixture was cooled and then added in ice-cold water $(15 \mathrm{~mL})$, stirred for further 30 minutes at room temperature. The water layer was extracted with chloroform $(3 \times 75 \mathrm{~mL})$ and organic layer were dried over sodium sulfate. It was concentrated under vacuum afforded solid was further recrystallized from chloroform: n-hexane (80:20) afforded compound 3 
in 70-75\% yields. This compound was characterized by IR, NMR data and compared with the literature M.P. $[15,16]$.

\section{(E)-N'-(3-cyano-4,5,6,7-tetrahydrobenzo [b]thiophen-2-yl)- $N$, $N$-dimethyl-formamidine (5)}

Compound $3(2.56 \mathrm{~g}, 0.01 \mathrm{~mol})$ and DMF-DMA $(1.32 \mathrm{~mL}, 0.01 \mathrm{~mol})$ in dry p-xylene $(15 \mathrm{~mL})$ was refluxed for $5 \mathrm{hr}$ (TLC checked, chloroform: methanol 9:1v/v). The excess of solvent was removed under reduced pressure. The solid obtained was stirred in $20 \mathrm{~mL}$ hexane for $4 \mathrm{hr}$ and was filtered washed with cold methanol. It was recrystallized from toluene as pale yellowish brown solid.

Light brown solid, Yield 80\%, 4g, m.p. $130{ }^{\circ} \mathrm{C}$; IR (Platinum ATR) 2288 (CN), 2935 (CH), 1613 $(\mathrm{C}=\mathrm{N}), 1620(\mathrm{C}=\mathrm{C}) 2825\left(\mathrm{CH}_{3}\right) ;{ }^{1} \mathrm{H} \mathrm{NMR}\left(500 \mathrm{MHz}, \mathrm{CDCl}_{3}\right) \delta 1.82\left(\mathrm{t}, J=4 \mathrm{~Hz}, 2 \mathrm{H}, \mathrm{CH}_{2}\right), 2.56(\mathrm{t}, J=4 \mathrm{~Hz}$, $2 \mathrm{H}, \mathrm{CH}_{2}$ ), 7.66 (s , 1H , NHCH), 3.07 (s , 3H , $\left.\mathrm{CH}_{3}\right), \mathrm{C}_{12} \mathrm{H}_{15} \mathrm{~N}_{3} \mathrm{~S}$ (233.10) :Calcd C, 61.77; H, 6.48; N, 18.01; S, 13.74 Found C, 61.81; H, 6.45; N, 18.04; S, 13.70

\section{$N^{\prime}$-(3-cyano-4,5,6,7-tetrahydrobenzo[b]thiophen-2-yl)-2-((E)formamido)acetic acid, (7a)}

Compound $5(0.1 \mathrm{gm} 0.43 \mathrm{mmol})$ and glycine $(0.30 \mathrm{mg}, 0.43 \mathrm{mmol})$ in ethanol and 2-3 drops glacial acetic acid was refluxed for $8 \mathrm{hr}$ (TLC Checked ,Hexane :ethyl acetate, 8:2 v/v). The solid obtained was filtered and recrystallized from ethanol to afford compound $\mathbf{7 a}$.

Light brown solid, Yield 80\%, 0.91mg, m.p. 124 ${ }^{\circ} \mathrm{C}$; IR (Platinum ATR) $2198(\mathrm{CN}), 2935(\mathrm{CH}), 1766(\mathrm{C}=\mathrm{N})$, $1620(\mathrm{C}=\mathrm{C}) ;{ }^{1} \mathrm{H}$ NMR $\left(500 \mathrm{MHz}, \mathrm{CDCl}_{3}\right) \delta 1.85\left(\mathrm{t}, J=4 \mathrm{~Hz}, 2 \mathrm{H}, \mathrm{CH}_{2}\right), 2.59\left(\mathrm{t}, J=4 \mathrm{~Hz}, 2 \mathrm{H}, \mathrm{CH}_{2}\right), 7.67(\mathrm{~d}, 1 \mathrm{H}$, $\mathrm{NHCH}), 2.50\left(\mathrm{q}, 1 \mathrm{H}, \mathrm{NHCH}_{2}\right), 3.59$ (d, $\left.2 \mathrm{H} \mathrm{CH}_{2} \mathrm{NH},\right), 10.9$ (s, 1H, OH). $\mathrm{C}_{12} \mathrm{H}_{13} \mathrm{~N}_{3} \mathrm{O}_{2} \mathrm{~S}$ (263.32): Calcd. C, 54.74; H, 4.98; N, 15.96; O, 12.15; Found C, 54.78; H, 4.95; N, 15.92; O, 12.20

\section{(E)- $N$-(aryl) (3-cyano-4,5,6,7-tetrahydrobenzo[b]thiophen-2-yl)-formamidine derivatives (7b-h)}

Compound 5 (0.1 gm $0.43 \mathrm{mmol})$ and aromatic amines $\mathbf{6 b}-\mathbf{h}(0.43 \mathrm{mmol})$ in ethanol and 2-3 drops glacial acetic acid refluxed for $8 \mathrm{hr}$ (TLC Checked, Hexane: Ethyl acetate ,8:2 v/v). The solid obtained was filtered and recrystallized from ethanol afforded compound $\mathbf{7 b}$.

Similar procedure was employed for the synthesis of compounds (7c-h).

(E)- $\mathrm{N}$-Phenyl (3-cyano-4,5,6,7-tetrahydrobenzo[b]thiophen-2-yl) formamidine (7b)

Light yellow solid, Yield: 68\%, $0.82 \mathrm{mg}, \mathrm{m} . \mathrm{p} .52^{\circ} \mathrm{C}$; IR (Platinum ATR) $\mathrm{cm}^{-1}$ : $3471(\mathrm{NH}), 1523(\mathrm{C}=\mathrm{N})$, 2262(CN), $1648(\mathrm{C}=\mathrm{C}) \mathrm{cm}^{-1} ;{ }^{1} \mathrm{H}$ NMR $\left(500 \mathrm{MHz}, \mathrm{DMSO}-d_{6}\right): \delta 6.62(\mathrm{t}, 1 \mathrm{H}, \mathrm{CH}), 6.46-7.01(\mathrm{~d}, \mathrm{~J}=3 \mathrm{~Hz} 1 \mathrm{H}$, $\mathrm{CH}), 4.0(\mathrm{~d}, 1 \mathrm{H}, \mathrm{CH}), 2.56-1.82\left(\mathrm{t}, J=4 \mathrm{~Hz}, 4 \mathrm{H}, \mathrm{CH}_{2}\right), 7.50(\mathrm{~d}, 1 \mathrm{H}, \mathrm{CH}) . \mathrm{C}_{16} \mathrm{H}_{15} \mathrm{~N}_{3} \mathrm{~S}$ (281.38): Calcd C, 68.30; H, 5.37; N, 14.93; Found C, 68.25; H, 5.42; N, 14.90

(E)- $N$-(4-chlorophenyl)- $N^{\prime}$-(3-cyano-4,5,6,7-tetrahydrobenzo[b]thiophen-2-yl)formamidine (7c)

Faint yellow solid, Yield :62\%, $0.84 \mathrm{mg}$, m.p. $110^{\circ} \mathrm{C}$; IR (Platinum ATR) cm ${ }^{-1}: 2927(\mathrm{NH}), 1620(\mathrm{C}=\mathrm{N}), 2198$ $(\mathrm{CN}), 1599(\mathrm{C}=\mathrm{C}), 721(\mathrm{Cl}) ;{ }^{1} \mathrm{H}$ NMR $\left(500 \mathrm{MHz}, \mathrm{DMSO}-d_{6}\right): \delta 6.62(\mathrm{t}, 1 \mathrm{H}, \mathrm{CH}), 6.40-7.02(\mathrm{t}, \mathrm{J}=4 \mathrm{~Hz} 1 \mathrm{H}$, $\mathrm{CH}), 4.0(\mathrm{~d}, 1 \mathrm{H}, \mathrm{CH}), 2.56-1.82\left(\mathrm{t}, J=4 \mathrm{~Hz}, 4 \mathrm{H}, \mathrm{CH}_{2}\right), 7.50(\mathrm{~d}, 1 \mathrm{H}, \mathrm{CH}) . \mathrm{C}_{16} \mathrm{H}_{14} \mathrm{ClN}_{3} \mathrm{~S}(315.82)$ : Calcd C, $60.85 ; \mathrm{H}, 4.47 ; \mathrm{Cl}, 11.23 ; \mathrm{N}, 13.31$; Found C, 60.90; H, 4.42; Cl, $11.20 ; \mathrm{N}, 13.34$

(E)- $N$-(4-bromophenyl)- $N$ '-(3-cyano-4,5,6,7-tetrahydrobenzo[b]thiophen-2-yl)formamidine (7d)

Light orange solid, Yield 61\%, $0.95 \mathrm{mg}$, m.p. $110^{\circ} \mathrm{C}$; IR (Platinum ATR) cm ${ }^{-1}: 2927(\mathrm{NH}), 1620(\mathrm{C}=\mathrm{N}), 2198$ $(\mathrm{CN}), 1599(\mathrm{C}=\mathrm{C}), 702(\mathrm{Br}) \mathrm{cm}^{-1}$; ${ }^{1} \mathrm{H}$ NMR $\left(500 \mathrm{MHz}, \mathrm{DMSO}-d_{6}\right): \delta 6.62(\mathrm{t}, 1 \mathrm{H}, \mathrm{CH}), 6.40-7.02(\mathrm{t}, \mathrm{J}=4 \mathrm{~Hz} 1 \mathrm{H}$, $\mathrm{CH}), 4.0(\mathrm{~d}, 1 \mathrm{H}, \mathrm{CH}), 2.56-1.82\left(\mathrm{t}, J=4 \mathrm{~Hz}, 4 \mathrm{H}, \mathrm{CH}_{2}\right), 7.50(\mathrm{~d}, 1 \mathrm{H}, \mathrm{CH}) . \mathrm{C}_{16} \mathrm{H}_{14} \mathrm{BrN}_{3} \mathrm{~S}(360.27)$ : Calcd C, 53.34; H, 3.92; Br, 22.18; N, 11.66; Found C, 53.30 H, 3.87; Br, 22.23; N, 11.66

\section{$\boldsymbol{N}$-(4-nitrophenyl)(E)- $\boldsymbol{N}^{\prime}$-(3-cyano-4,5,6,7-tetrahydrobenzo[b]thiophen-2-yl)-formamidine (7e)}

Yellow solid, Yield 70\%, $0.98 \mathrm{mg}$, m.p. $95^{\circ} \mathrm{C}$; IR (Platinum ATR) cm ${ }^{-1}: 3425(\mathrm{NH}), 1620(\mathrm{C}=\mathrm{N}), 2175(\mathrm{CN})$, $1557\left(\mathrm{NO}_{2}\right), 1470(\mathrm{CH}) \mathrm{cm}^{-1} ;{ }^{1} \mathrm{H}$ NMR $\left(500 \mathrm{MHz}, \mathrm{DMSO}-d_{6}\right): \delta 6.72(\mathrm{~d}, 1 \mathrm{H}, \mathrm{CH}), 6.72-7.94(\mathrm{t}, \mathrm{J}=7 \mathrm{~Hz} 1 \mathrm{H}$, $\mathrm{CH}), 4.0(\mathrm{~d}, 1 \mathrm{H}, \mathrm{CH}), 2.56-1.82\left(\mathrm{t}, J=4 \mathrm{~Hz}, 4 \mathrm{H}, \mathrm{CH}_{2}\right), 7.50(\mathrm{~d}, 1 \mathrm{H}, \mathrm{CH}) . \mathrm{C}_{16} \mathrm{H}_{14} \mathrm{~N}_{4} \mathrm{O}_{2} \mathrm{~S}(326.37)$ : Calcd C, 58.88; H, 4.32; N, 17.17; O, 9.80; Found C, 58.80; H, 4.40; N, 17.17; O, 9.88

(E)- $N$-(3-chlorophenyl)- $N^{\prime}$-(3-cyano-4,5,6,7-tetrahydrobenzo[b]thiophen-2-yl)formamidine (7f) Light yellow solid, Yield $60 \%, 0.82 \mathrm{mg}$, m.p. $110^{\circ} \mathrm{C}$; IR (Platinum ATR) $\mathrm{cm}^{-1}: 2927(\mathrm{NH}), 1620(\mathrm{C}=\mathrm{N}), 2198$ $(\mathrm{CN}), 1599(\mathrm{C}=\mathrm{C}), 721(\mathrm{Cl}) \mathrm{cm}^{-1} ;{ }^{1} \mathrm{H}$ NMR (500 MHz, DMSO-d $): \delta 6.47(\mathrm{~s}, 1 \mathrm{H}, \mathrm{CH}), 6.63(\mathrm{~d}, 1 \mathrm{H}, \mathrm{CH})$, 6.34(d,1H,CH), 6.95(d, 1H,CH) $4.0(\mathrm{~d}, 1 \mathrm{H}, \mathrm{CH}), 2.56-1.82\left(\mathrm{t}, \mathrm{J}=4 \mathrm{~Hz}, 4 \mathrm{H}, \mathrm{CH}_{2}\right), 7.50(\mathrm{~d}, 1 \mathrm{H}, \mathrm{CH})$. $\mathrm{C}_{16} \mathrm{H}_{14} \mathrm{ClN}_{3} \mathrm{~S}$ (315.82): Calcd C, 60.85; H, 4.47; Cl, 11.23; N, 13.31; Found C, 60.82; H, 4.35; Cl, 11.28; N, 13.37 
(E)- $N$-(3-nitrophenyl)- $N$ '-(3-cyano-4,5,6,7-tetrahydrobenzo[b]thiophen-2-yl)formamidine (7g)

Light yellow solid, Yield 60\%, $106 \mathrm{mg}$, m.p. $110^{\circ} \mathrm{C}$; IR (Platinum ATR) $\mathrm{cm}^{-1}: 3425(\mathrm{NH}), 1620(\mathrm{C}=\mathrm{N}), 2175$ $(\mathrm{CN}), 1557\left(\mathrm{NO}_{2}\right), 1470(\mathrm{CH}) \mathrm{cm}^{-1} ;{ }^{1} \mathrm{H}$ NMR $\left(500 \mathrm{MHz}, \mathrm{DMSO}-d_{6}\right): \delta 6.75(\mathrm{~d}, 1 \mathrm{H}, \mathrm{CH}), 7.22(\mathrm{t}, 1 \mathrm{H}, \mathrm{CH})$, 7.45(t,1H,CH), 7.30(s, $1 \mathrm{H}, \mathrm{CH}), 4.0(\mathrm{~d}, 1 \mathrm{H}, \mathrm{CH}), 2.56-1.82\left(\mathrm{t}, J=4 \mathrm{~Hz}, 4 \mathrm{H}, \mathrm{CH}_{2}\right), 7.50(\mathrm{~d}, 1 \mathrm{H}, \mathrm{CH})$. $\mathrm{C}_{16} \mathrm{H}_{14} \mathrm{~N}_{4} \mathrm{O}_{2} \mathrm{~S}$ (326.37): Calcd. C, 58.88; H, 4.32; N, 17.17; O, 9.80; Found C, 58.70; H, 4.45; N, 17.17; O, 9.83

\section{$N$-pyridin (E)-N'-(3-cyano-4,5,6,7-tetrahydrobenzo[b]thiophen-2-yl)formamidine (7h)}

Yellowish brown solid, Yield: $68 \%, 0.83 \mathrm{mg}$, m.p. $120^{\circ} \mathrm{C}$; IR (Platinum ATR) $\mathrm{cm}^{-1}: 1620(\mathrm{NH}), 1573(\mathrm{C}=\mathrm{N})$, $2198(\mathrm{CN}), \mathrm{cm}^{-1} ;{ }^{1} \mathrm{H}$ NMR $\left(500 \mathrm{MHz}, \mathrm{DMSO}-d_{6}\right): \delta 6.70(\mathrm{~d}, 1 \mathrm{H}, \mathrm{CH}), 7.44(\mathrm{t}, 1 \mathrm{H}, \mathrm{CH}), 6.60(\mathrm{t}, 1 \mathrm{H}, \mathrm{CH}), 8.11(\mathrm{~d}$ , $1 \mathrm{H}, \mathrm{CH}), 4.0(\mathrm{~d}, 1 \mathrm{H}, \mathrm{CH}), 2.56-1.82\left(\mathrm{t}, J=4 \mathrm{~Hz}, 4 \mathrm{H}, \mathrm{CH}_{2}\right), 7.50(\mathrm{~d}, 1 \mathrm{H}, \mathrm{CH}) ; \mathrm{C}_{15} \mathrm{H}_{14} \mathrm{~N}_{4} \mathrm{~S}(282.36):$ Calcd $\mathrm{C}$, 63.80; H, 5.00; N, 19.84; Found C, 63.69; H, 5.11; N, 19.90

\section{Conclusion}

We have successfully prepared (E)-N'-(3-cyano-4,5,6,7-tetrahydrobenzo [b]thiophen-2-yl)- $N, N$ dimethylformamidine derivatives and structures were confirmed spectral data and analytical data. The compound $\mathbf{7 c}$ and $\mathbf{7 d}$ showed moderate while $\mathbf{7 g}$ showed excellent antibacterial activity. Also compound $\mathbf{7 c}, \mathbf{7 d}$ and $7 \mathbf{e}$ showed excellent antifungal activity.

\section{Acknowledgements}

The authors are thankful CIF, Savitribai Phule Pune University, Pune and SAIF, Punjab University, Chandigarh for spectral analysis, M.V.P. Samaj and Principal, K.R.T. Arts, B.H. Commerce and A. M. Science College, Shivajinagar, Gangapur Road, Nashik- 422 002, (MS), India for facilities.

\section{References}

[1] Puterová, Z., Krutoíková, A. and Végh, D., Arkivoc, 2010 (i), 209.

[2] Balamurugan, K., Perumal, S., Reddy, A. S. K., Yogeeswari, P., Sriram, D., Tetrahedron Lett., 50, $2009,6191$.

[3] Fakhr, I. M. I., Radwan, M. A. A., El-Batran, S., Omar, M. E., El-Salam, A. and El-Shenawy, S. M., Eur. J. Med. Chem., 44, $2009,1718$.

[4] Isloor, A. M., Kalluraya, B. and Pai, K. S., Eur. J. Med. Chem., 45, 2010,825.

[5] Amr, A. E. G. E., Sherif, M. H., Assy, M. G., Al-Omar, M. A. and Raga, I., Eur. J. Med. Chem., 45, $2010,5935$.

[6] Gütschow, M., Kuerschner, L., Neumann, U., Pietsch, M., Löser, R., Koglin, N. and Eger, K., J. Med. Chem., $42,1999,5437$.

[7] Pinkerton, A. B., Lee, T. T., Hoffman, T. Z., Wang, Y., Kahraman,M., Cook, T. G., Severance, D., Gahman, T. C., Noble, S. A.,Shiau, A. K. and Davis, R. I., Bioorg. Med. Chem. Lett., 17, 2007, 3562.

[8] Romagnoli, R., Baraldi, P. G., Pavani, M. G., Tabrizi, M. A., Preti, D., Fruttarolo, F., Piccagli, L., Jung, M. K., Hamel, E., Borgatti, M. and Gambari, R., J. Med. Chem., 49 (13), 2006,3906.

[9] Ravindranathan, K. P., Mandiyan, V., Ekkati, A. R., Bae, J. H., Schlessinger, J. and Jorgensen, W. L., J. Med. Chem., 53, $2010,1662$.

[10] Aurelio, L., Valant, C., Figler, H., Flynn, B. L., Linden, J.,Sexton, P. M., Christopoulos, A. and Scammells, P. J., Bioorg. Med. Chem. 17 (20), 2009,7353.

[11] K. Madhavi, K. V. Ramanamma Int.J.Curr.Microbiol.App.Sci 5(1), 2016, 364-375

[12] Magdy Mohamed Hemdan, Heba Kamal Abd El-Mawgoude. Chem. Pharm. Bull. 63, 2015,812-818

[13] Sajal Srivastava, Barnali Das. Der Pharma Chemica, 3 (6), 2011, 103-111

[14] Hend Nagah Hafez, Omar Khalid Al-Duaij, Abdel-Rhman Barakat A. El-Gazzar. Int J Org Chem, 3, $2013,110-118$.

[15] Mohamed Said and Hosam Elshihawy. Pak. J. Pharm. Sci., 27(4), 2014,885-892

[16] Pinto, I.L., Jarvest, R.L. and serafinowska, H.T., Tetrahedron Lett., 41, 2000,1597.

[17] Baraldi, P. G., Pavani, M. G., Shryock, J. C., Moorman, A. R., Iannotta, V., Borea, P. A. and Romagnoli, R., Eur. J. Med. Chem., $39,2004,855$.

[18] Shams, H. Z., Elkholy, Y. M., Azzam, R, A. and Mohareb, R. M., Phosph. Sulf. Silicon., 155, $1999,215$.

[19] Shams, H. Z., Mohareb, R. M., Helal, M. H. and Mahmoud, A. E., Phosph. Sulf. Silicon., 182, $2007,237$.

[20] Sherif, S. M., Mohareb, R. M., Shams, H. Z. and Gaber, H. M., J. Chem. Res., (S), 434; (M) 1995,2658.

[21] Hoda Z. S., Rafat M. M., Maher H. H. and Amira E. M.,Molecules, 16, 2011, 52.

[22] Hoda Z. S., Rafat M. M., Maher H. H. and Amira E. M.,Molecules, 16, 2011,6271.

[23] Gewald, K. and Schindler, R., J. Prakt. Chem. 332, 1990,223. b. Gewald, K.; Elfriede, S.; B. Horst, Chem. Ber. $99,1966,94$.

[24] Huang, W.; Li, J. Synth. Comm35, 2005,1351.

[25] Sabnis, R. W.; Rangnekar, D. W.; Sonawane, N. D.; J Het Chem. 36, 1999, 333. 